\title{
Transition to coarsening for confined one-dimensional interfaces with bending rigidity
}

\author{
Thomas Le Goff ${ }^{1}$, Paolo Politi ${ }^{2,3}$ and Olivier Pierre-Louis ${ }^{1 *}$ \\ ${ }^{1}$ Institut Lumière Matière, UMR5306 Université Lyon 1-CNRS, Université de Lyon 69622 Villeurbanne, France \\ ${ }^{2}$ Istituto dei Sistemi Complessi, Consiglio Nazionale delle Ricerche, \\ Via Madonna del Piano 10, 50019 Sesto Fiorentino, Italy and \\ ${ }^{3}$ INFN Sezione di Firenze, via G. Sansone 1, 50019 Sesto Fiorentino, Italy
}

(Dated: October 26, 2018)

\begin{abstract}
We discuss the nonlinear dynamics and fluctuations of interfaces with bending rigidity under the competing attractions of two walls with arbitrary permeabilities. This system mimics the dynamics of confined membranes. We use a two-dimension hydrodynamic model, where membranes are effectively one-dimensional objects. In a previous work [T. Le Goff et al, Phys. Rev. E 90, 032114 (2014)], we have shown that this model predicts frozen states caused by bending rigidity-induced oscillatory interactions between kinks (or domain walls). We here demonstrate that in the presence of tension, potential asymmetry, or thermal noise, there is a finite threshold above which frozen states disappear, and perpetual coarsening is restored. Depending on the driving force, the transition to coarsening exhibits different scenarios. First, for membranes under tension, small tensions can only lead to transient coarsening or partial disordering, while above a finite threshold, membrane oscillations disappear and perpetual coarsening is found. Second, potential asymmetry is relevant in the non-conserved case only, i.e. for permeable walls, where it induces a drift force on the kinks, leading to a fast coarsening process via kink-antikink annihilation. However, below some threshold, the drift force can be balanced by the oscillatory interactions between kinks, and frozen adhesion patches can still be observed. Finally, at long times, noise restores coarsening with standard exponents depending on the permeability of the walls. However, the typical time for the appearance of coarsening exhibits an Arrhenius form. As a consequence, a finite noise amplitude is needed in order to observe coarsening in observable time.
\end{abstract}

\section{INTRODUCTION}

Bending rigidity is a crucial ingredient in soft matter systems, which leads to a number of nontrivial effects in the equilibrium and non-equilibrium behavior of membranes [1, 2] and filaments 3 5]. For example, in equilibrium, the minimization of bending energy leads to non-trivial shapes of membrane vesicles [6], and knotted filaments [7]. In non-equilibrium conditions, bending rigidity also plays a crucial role in cell adhesion, or in the rheology and stability of membrane stacks [8, 9].

Here we explore the dynamical behavior of a model where an interface with bending rigidity is confined between two walls. This model aims at understanding the behavior of lipid membranes confined into doublewell potentials. Such double well potentials have been evidenced in various experimental contexts, for example when a membrane is placed under the combined influences of a short-range attraction induced by ligandreceptor pairs, and a medium range repulsion caused by polymer brushes mimicking glycocalyx [10, 11. A second example is that of intermembrane attraction in the presence of ligand-receptor pairs with two different lengths [12. We also expect this double-well picture to represent membrane adhesion to the cytoskeleton or to a substrate during cell adhesion. Indeed, cell membranes are known to be able to bind to, or unbind from the cytoskeletal cortex during the adhesion of cells to a substrate, e.g. to form blebs [13 15].

\footnotetext{
* olivier.pierre-louis@univ-lyon1.fr
}

For the sake of simplicity, and following our previous work [16], we consider a two-dimensional system. As a consequence, the interface - hereafter denoted as a membrane - is effectively a one-dimensional object. The walls attract the membrane, mimicking physical adhesion 9, 17, 18 (such as Van der Waals interactions, hydration interactions, osmotic pressures, and entropic interactions), or specific adhesion [19, 20] via a simple effective adhesion potential. We have recently shown that in such a model the membrane bending rigidity leads to arrested dynamics 16, with frozen adhesion patches on both walls.

Other studies in the literature have suggested that finite-size adhesion patches may also be induced by more complex (bio)physical ingredients, such as the clustering ligand-receptor pairs [21 23], the disorder of the environment 24], the trapping of ligands in membrane partitions 25, or the active remodeling of the cytoskeleton [26]. One aim of our simple modeling is to provide hints towards a better understanding of the formation of finite-size adhesion patches.

Moreover, the spatial organization of the frozen states observed in Ref. [16] was strongly influenced by the permeability of the confining walls. Indeed, strong wall permeabilities were shown to give rise to disordered states, while vanishing permeabilities lead to ordered states with a periodic arrangement of patches [16.

Here, we discuss the robustness of the frozen states with respect to various physical "perturbations": membrane tension, potential asymmetry, and thermal fluctuations. We find that in all cases, the frozen states can be destroyed, and coarsening can be restored when the 
amplitude of these effects exceeds a finite threshold.

In the following, we start in Section II with the derivation of the evolution equation for the membrane in the lubrication limit. We focus on the two opposite regimes of very permeable, and perfectly impermeable walls, respectively leading to non-conserved and conserved dynamics. We then report the equations of quantitative kink model derived in Ref. 27. We also recall the main results regarding the existence of frozen states [16 in Section III.

In Section IV] we show that there is a critical tension $\sigma_{c}$ above which the oscillations of the membrane profile disappear. In this regime, the resulting dynamics is similar to that of the standard Time-Dependent GinzburgLandau (TDGL) equation 28 for the permeable case, and Cahn-Hilliard (CH) equation [28, 29, for the impermeable case, with perpetual coarsening caused by attractive interactions between neighboring kinks. For finite tensions below the threshold $\sigma_{c}$, one can observe transient coarsening (i.e. which stops after some finite time). This transient coarsening is able to alter the perfect order observed in the conserved case in the absence of tension.

Then, in Section V] we discuss the consequences of an asymmetric adhesion potential, favoring the adhesion on one of the two walls. While it is irrelevant in the conserved case, this asymmetry gives rise to a drift force on the kinks in the non-conserved case. This drift tends to increase the size of the adhesion patches on the favored wall. When the asymmetry is large enough, the resulting drift leads an enhanced kink-antikink collision and annihilation rate, giving rise to a fast coarsening scenario with a final state where the membrane is only on the side of the favored wall. However, for weak asymmetries, the drift force on the kinks is not strong enough to overcome kink interactions. Thus, localized frozen adhesion patches can still be found.

Furthermore, the effect of thermal noise is analyzed in Section VI, using the kink model supplemented with Langevin forces. The results indicate that noise always lead to perpetual coarsening. However, the time for the appearance of coarsening exhibits and Arrhenius law. Hence, observable coarsening in a finite time requires a finite noise amplitude, i.e. a finite temperature.

Finally, we conclude in Section VII.

\section{MEMBRANE LUBRICATION MODEL}

Assuming for simplicity a two-dimensional system in the $z, x$ plane, we wish to describe the dynamical behavior of a lipid membrane of height $z=h(x, t)$. This membrane is confined between two walls at $z= \pm h_{0}$, as shown in Figure (1), and is surrounded by an incompressible fluid in the Stokes regime, obeying

$$
\nabla p-\mu \nabla^{2} \mathbf{v}=0 .
$$

Here, $p$ is the pressure, $\mu$ the viscosity, and $\mathbf{v}$ is the fluid velocity. The membrane is assumed to be impermeable. Moreover we consider a no slip condition at the walls,

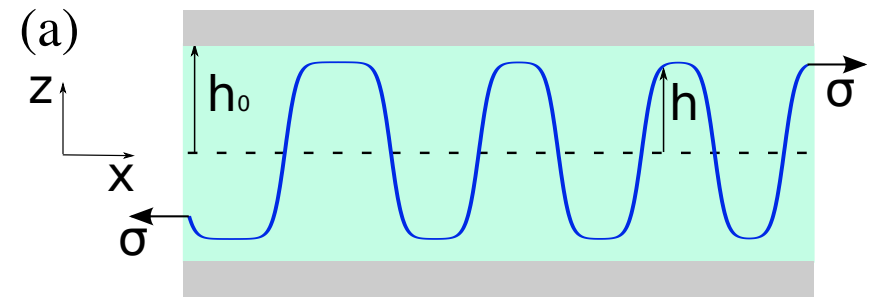

(b)
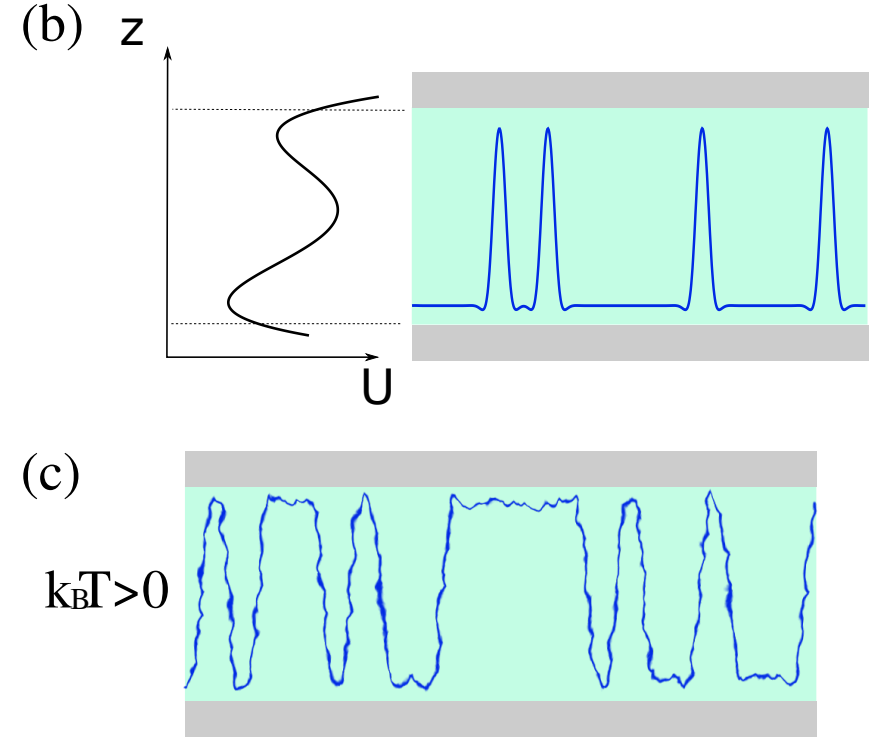

FIG. 1. Schematic of a confined membrane. The wall on the top is at $z=h_{0}$, and the bottom wall is at $z=-h_{0}$. The blue solid line at $h(x, t)$ is the height of the membrane. Quantities above the membrane are written with + and those below with -. We discuss the influence of three physical ingredients on the dynamics of confined membranes (a) a tension $\sigma$, (b) an asymmetry of the adhesion potential $\mathcal{U}(h)$, and (c) thermal fluctuations.

leading to $v_{x}\left( \pm h_{0}\right)=0$. Arbitrary wall permeability is accounted for using a phenomenological kinetic law

$$
v_{z \pm}\left( \pm h_{0}\right)= \pm \nu\left(p_{ \pm}-p_{\text {ext }}\right),
$$

where $\nu$ is permeability kinetic coefficient, $p_{ \pm}$is the pressure at $z= \pm h_{0}$, and $p_{\text {ext }}$ is a constant external pressure.

Here, we consider both membrane bending rigidity and membrane tension via the standard Helfrich model [1, 2]. The membrane is also subject to a double well potential $\mathcal{U}(h)$, which accounts for its interaction with the walls. In the small slope limit, which is discussed below, the total energy of the membrane is

$$
\mathcal{E}=\int \mathrm{d} x\left(\frac{\kappa}{2}\left(\partial_{x x} h\right)^{2}+\frac{\sigma}{2}\left(\partial_{x} h\right)^{2}+\mathcal{U}(h)\right) .
$$

where $\partial_{x}$ denotes the partial derivative with respect to $x$, $\kappa$ is the bending rigidity modulus, and $\sigma$ is the tension.

Following Ref. [16, we consider the lubrication regime, where the horizontal scale is much bigger than vertical scale, i.e. $\partial_{x} h \ll 1$ with $h \sim h_{0}$. In this limit, the hydrodynamic flow is to leading order along $x$, with a parabolic Poiseuille-like profile along $z$. In addition, we 
focus on the two limits of very permeable walls $\nu \rightarrow \infty$, and impermeable walls $\nu \rightarrow 0$, respectively leading to

$$
\begin{aligned}
& \partial_{t} h=\frac{\nu}{2} f_{z}, \\
& \partial_{t} h=\partial_{x}\left[-\frac{h_{0}^{3}}{24 \mu}\left(1-\frac{h^{2}}{h_{0}^{2}}\right)^{3} \partial_{x} f_{z}\right],
\end{aligned}
$$

with the force along $z$ acting on the membrane

$$
f_{z}=-\kappa \partial_{x}^{4} h+\sigma \partial_{x x} h-\mathcal{U}^{\prime}(h) .
$$

As discussed in Ref. [16, in the impermeable case there are additional nonlocal terms. However, these terms are irrelevant for dynamics. We can therefore safely neglect them. For the sake of simplicity we also neglect the nonlinear mobility term $\left(1-h^{2} / h_{0}^{2}\right)^{3}$ appearing in the conserved case. Its main consequence is to slow down the dynamics in the late stages by a constant factor. Finally, in rescaled coordinates, Eqs. (45) now read

$$
\begin{aligned}
& \partial_{T} H=-\partial_{X}^{4} H+\gamma \partial_{X X} H-U^{\prime}(H), \\
& \partial_{T} H=\partial_{X X}\left(\partial_{X}^{4} H-\gamma \partial_{X X} H+U^{\prime}(H)\right),
\end{aligned}
$$

with $X=\left[\mathcal{U}_{0} /\left(\kappa h_{0}^{2}\right)\right]^{1 / 4} x$ and $H=h / h_{0}$. The normalized time variable is $T=t \nu \mathcal{U}_{0} /\left(2 h_{0}^{2}\right)$ in the non-conserved permeable case, and $T=\mathcal{U}_{0}^{3 / 2} t /\left(24 \mu \kappa^{1 / 2}\right)$ in the conserved impermeable case. We have also defined the normalized tension:

$$
\gamma=\frac{h_{0} \sigma}{\kappa^{1 / 2} \mathcal{U}_{0}^{1 / 2}} .
$$

Using the parameter $\gamma$, two limits can be defined. First, when $\gamma \gg 1$, i.e. $\kappa \rightarrow 0$, the fourth order derivative in the force term in Eqs. 778 is negligible, and one recovers the standard Time Dependent Ginzburg Landau (TDGL) equation, and Cahn Hilliard $(\mathrm{CH})$ equation respectively. In the opposite case $\gamma \ll 1$, i.e. $\sigma \rightarrow 0$, the second order derivative in the force term in Eqs. (7/8) is negligible. We denote the resulting equations as the TDGL4 and CH4 equations respectively.

An additional level of coarse graining is possible, based on the dynamics of kinks, which are defined as the transition zones, or domain walls, separating adhesion patches in the two different wells of the potential $U$. Kink dynamics have been derived in the 80s for the TDGL and $\mathrm{CH}$ equations [30, 31]. Following our recent generalized derivation of kink dynamics including bending rigidity [27, and a symmetric potential $(U(-H)=U(H))$ the position $X_{n}$ of the $n$th kink obeys:

$$
\begin{aligned}
\dot{X}_{n}= & \frac{1}{B_{1}} \Delta \tilde{R}_{n}, \\
\dot{X}_{n}= & \frac{1}{B_{0}^{2} \ell_{n-1 / 2} \ell_{n+1 / 2}-B_{2}\left(\ell_{n-1 / 2}+\ell_{n+1 / 2}\right)}\{ \\
& \ell_{n-1 / 2}\left(B_{2} \dot{X}_{n+1}+\tilde{R}_{n+3 / 2}-\tilde{R}_{n-1 / 2}\right) \\
& \left.+\ell_{n+1 / 2}\left(B_{2} \dot{X}_{n-1}+\tilde{R}_{n+1 / 2}-\tilde{R}_{n-3 / 2}\right)\right\}
\end{aligned}
$$

for permeable and impermeable walls, respectively. above the most important quantity is the function $\tilde{R}(\ell)$, derived in Ref. 27, which is exponentially decreasing and possibly oscillating for large $\ell$. Its explicit form will be given later. As for notations, we have defined the difference operator $\Delta$ such that $\Delta Y_{n}=Y_{n+1 / 2}-Y_{n-1 / 2}$ for any quantity $Y_{n}$. Moreover, the inter-kink distance is denoted as $\ell_{n+1 / 2}=X_{n+1}-X_{n}=\Delta X_{n+1 / 2}$, and we also use the notation $\tilde{R}_{n+1 / 2}=\tilde{R}\left(\ell_{n+1 / 2}\right)$. Finally, the constants

$$
\begin{aligned}
B_{0} & =H_{k}(+\infty)-H_{k}(-\infty) \\
B_{1} & =\int_{-\infty}^{+\infty} \mathrm{d} X\left(\partial_{X} H_{k}\right)^{2} \\
B_{2} & =\int_{-\infty}^{\infty} \mathrm{d} X\left[H_{k}(+\infty)-H_{k}(X)\right]\left[H_{k}(X)-H_{k}(-\infty)\right]
\end{aligned}
$$

are calculated from the profile $H_{k}(X)$ of an isolated kink. Notice that $B_{0}$ is simply the distance between the two minima of the double well potential. $B_{0}>0$ for kinks, and $B_{0}<0$ for antikinks. In addition, we have $B_{1}>$ 0 . Moreover, $B_{2}>0$ is positive for all monotonically varying kink profiles. For more complex profiles, the sign of $B_{2}$ is not know a priori, but for all cases discussed below $B_{2}>0$.

The implementation of the kink dynamics provides an analytically simplified - and numerically lighter - way to compute membrane dynamics. The quantitative accuracy of these equations was tested and confirmed in Ref. 27] from a direct integration of the full dynamics. Kink dynamics is asymptotically exact when the distance between kinks is large.

\section{THE BASIC MODEL: FROZEN STATES AND ORDER-DISORDER TRANSITION}

In Ref. 16 we have studied the dynamics emerging from Eqs. (78) in the absence of tension $\sigma=0$, for a symmetric potential $\mathcal{U}(h)=\mathcal{U}(-h)$, and without fluctuations. Let us recall the main results.

First, both in the permeable and impermeable cases, dynamics are rapidly arrested and the membrane profile reaches a frozen steady-state. The origin of this steadystate was traced back to the presence of oscillatory interactions between kinks, which are caused by the bending rigidity.

Using typical orders of magnitude with a physical potential including hydration repulsion and van der Waals attraction [17, we find that the scale $L$ of the adhesion patches is [16] $L \sim h_{0}^{1 / 2} \kappa^{1 / 4} \mathcal{U}_{0}^{-1 / 4}$. Using $h_{0} \approx 10$ to $20 \mathrm{~nm}$ as suggested by experiments in Refs. 10, 12, typical adhesion patch lengths are predicted to range from $100 \mathrm{~nm}$ to $1 \mu \mathrm{m}$. Since the slopes are bounded at all times in the rescaled coordinates, they remain small in physical coordinates, and the lubrication approximation is self-consistent. 
The second main result of Ref. [16] is that, starting from small random initial conditions, very permeable or impermeable walls respectively lead to disordered or ordered frozen configurations. In order to understand this result, we first recall that the membrane is initially destabilized by the competitive attractions of the two walls in opposite directions. Since short wavelength perturbations are stabilized by bending rigidity, the instability can only be present at long enough wavelengths. For permeable walls, modes of large wavelength all have the same dissipation rate, which is essentially that of the translation of a flat membrane in the $z$ direction. As a consequence, all the long wavelength modes have the same growth rate, and many wavelengths are present simultaneously, leading to a disordered membrane profile. However, for impermeable walls, this translational mode along $z$ is forbidden due to mass conservation, and the increase of the amplitude of long wavelengths modes require flows along $x$ over large scales which are impeded due to their large cost in viscous dissipation. Since long wavelength perturbations are slowed down, and small wavelength perturbations are stable, an optimum wavelength exists and the instability develops at some welldefined intermediate scale, leading to an ordered periodic state.

This result is readily obtained from the linear stability analysis of Eqs.(78). Indeed, assuming small perturbations of amplitude $\epsilon \ll 1$, and wavelength $\lambda$ around a flat profile $H=\bar{H}+\epsilon \exp i(\omega T+q X)$, with $q=2 \pi / \lambda$, one obtains the following dispersion relation:

$$
i \omega=-A_{q}\left[q^{4}+\gamma q^{2}+U^{\prime \prime}(\bar{H})\right],
$$

where we have defined $A_{q}=1$ for the non-conserved case and $A_{q}=q^{2}$ for the conserved case. A positive real part of the growth rate $i \omega$ indicates an instability. Thus, the membrane is unstable when $U^{\prime \prime}(\bar{H}) \leq 0$. In addition, we confirm that all long wavelength perturbations grow with the same growth rate in the case of TDGL4. In contrast, the growth rate $i \omega$ exhibits a maximum for $\mathrm{CH} 4$.

From the analysis of the periodic nonlinear steadystates, we have further shown in Ref. [16] that the periodic pattern emerging in the impermeable case are stable, so that no further evolution is possible and the membrane profile remains frozen in this ordered state. In the nonconserved case, the disordered pattern emerging from the linear instability rearranges, and reaches a frozen disordered steady-state [16].

We have now finished to review the basic model. The next three Sections report original results on the effects of membrane tension (Sec. IV), asymmetric potential (Sec. V), and noise (Sec. VI).

\section{FINITE MEMBRANE TENSION}

Even though bending rigidity plays a major role in membrane dynamics, experiments usually report the existence of an effective tension, varying from $10^{-5}$ to $10^{-3}$
$\mathrm{Jm}^{-2}$ [9, 17, 32]. Therefore, in this section we wish to discuss its effect.

As already noticed in Ref. [16, the tension-dominated limit where $\kappa=0$ and $\sigma \neq 0$ leads to the standard TDGL and $\mathrm{CH}$ models respectively for permeable and impermeable walls, which exhibit monotonous kink profiles leading to attractive non-oscillatory interaction between kinks, which trigger perpetual coarsening. From these results, it is natural to investigate the dynamics at finite values of $\sigma$ and $\kappa$, mainly for two reasons. First, we aim to identify the threshold above which coarsening can be observed. Second, even when no perpetual coarsening is present, we point out that tension is able to affect the spatial organization of the frozen states observed at $\sigma=0$.

\section{A. Critical tension}

As discussed above, a crucial property of Eqs. $(78)$ is the existence of oscillations in the kink tails, at the edges of adhesion patches. Consider a small perturbation $H=$ $H_{m}+\delta H$, where $H_{m}$ is a minimum of the potential $U$. To leading order, both in the conserved and non-conserved cases, stationary states obey

$$
\partial_{X}^{4} \delta H-\gamma \partial_{X X} \delta H+U_{m}^{\prime \prime} \delta H=0,
$$

where $U_{m}^{\prime \prime}=U^{\prime \prime}\left(H_{m}\right)$. Assuming $\delta H \sim \exp (-r X)$, we obtain

$$
r^{4}-\gamma r^{2}+U_{m}^{\prime \prime}=0
$$

Solving Eq. 177 with the two constraints: $U_{m}^{\prime \prime}>0$, and $\gamma>0$, we find two different regimes separated by the critical tension

$$
\gamma_{c}=\left(4 U_{m}^{\prime \prime}\right)^{1 / 2} .
$$

For small normalized tensions $\gamma<\gamma_{c}$ the oscillations are still present. Considering only the profiles $\delta H(X)=$ $R(X)$ decaying for $X \rightarrow+\infty$ (profiles decaying as $X \rightarrow$ $-\infty$ can be obtained by symmetry), we find

$$
R(X)=A \cos \left(Q_{1} X+\alpha\right) \exp \left(-Q_{2} X\right) .
$$

where

$$
Q_{1,2}=\left[\left(U_{m}^{\prime \prime 1 / 2}\right) / 2 \mp \gamma / 4\right]^{1 / 2} .
$$

Hence, the wavelength of the oscillation $\Lambda=2 \pi / Q_{1}$ increases when the tension increases. At the threshold, $\Lambda$ diverges and the kink profile becomes monotonic.

For larger normalized tensions $\gamma>\gamma_{c}$, the profile is the superposition of two non-oscillating exponentials:

$$
R(X)=A_{+} \exp \left(-Q_{+} X\right)+A_{-} \exp \left(-Q_{-} X\right),
$$

where

$$
Q_{ \pm}=2^{-1 / 2}\left[\gamma \pm\left(\gamma^{2}-4 U_{m}^{\prime \prime}\right)^{1 / 2}\right]^{1 / 2}
$$


Following Ref. [27, the function $\tilde{R}$ which intervenes in the evolution equation (10 11) for the kink positions, is obtained from $R$ as follows:

$$
\tilde{R}(X)=2\left[U_{m}^{\prime \prime} R^{2}\left(\frac{X}{2}\right)-R^{\prime \prime 2}\left(\frac{X}{2}\right)\right] .
$$

Hence, $\tilde{R}$ is oscillatory when $R$ is oscillatory. As a consequence of the disappearance of oscillatory kink tails in $R$ and $\tilde{R}$, we expect that coarsening should be restored for $\gamma>\gamma_{c}$.

In order to check this prediction, we have obtained numerical solutions of Eqs. (7/8) in the presence of tension with an initial condition consisting of small perturbations around the average height $\bar{H}=0$. We choose the specific potential

$$
U(H)=\frac{1}{4}\left(H_{m}^{2}-H^{2}\right)^{2},
$$

with $H_{m}=0.9$, leading to the critical tension $\gamma_{c} \simeq 2.55$. The existence of this threshold is confirmed both in the conserved and non-conserved cases. As shown in Figs. 2[3, coarsening is stopped for $\gamma=2$, while perpetual coarsening is observed for $\gamma=3$. While the full membrane dynamics Eq. (7) can be implemented for the non-conserved case, we have used kink dynamics Eq.(11) to reach long enough time-scales in the conserved case. For $\gamma=3>\gamma_{c}$, we have implemented the kink dynamics using only the exponential contribution $Q_{+}$in Eq. 21. Indeed, due to its slower decay, the term involving $Q_{+}$is always be dominant at large scales.

\section{B. Transient coarsening and disordering in $\mathrm{CH} 4$}

In Fig. 3, we also see that, for conserved dynamics in the presence of a small tension $\gamma=2<\gamma_{c}$, a finite amount of coarsening can be observed. Moreover, in contrast to the tensionless case, some disorder is obtained in the final configuration.

To understand this result, we perform a straightforward extension of the results of Eq.(23) in Ref. [16] on the stability of periodic steady-states to the case of finite tensions. We obtain that periodic steady-states are unstable if $\partial_{\lambda} \mathcal{L}_{\lambda} \leq 0$, and stable if $\partial_{\lambda} \mathcal{L}_{\lambda} \geq 0$, with

$$
\mathcal{L}_{\lambda}=-\int_{0}^{\lambda} \mathrm{d} X\left[2\left(\partial_{X X} H\right)^{2}+\gamma\left(\partial_{X} H\right)^{2}\right]
$$

As seen on Fig. 4, this criterion reveals that the periodic state of wavelength $\lambda_{m} \simeq 14.87$ emerging from the linear instability is unstable, i.e. $\partial_{\lambda} \mathcal{L}_{\lambda_{m}} \leq 0$. This is in contrast with the tensionless limit where the linear instability was producing a stable periodic steady-state [16].

As a consequence of the unstable character of the periodic steady-state with $\lambda \simeq \lambda_{m}$, the system reorganizes into a non-periodic state with a larger wavelength, as seen in the histogram in Fig. 4. Then, the coarsening stops, and the membrane profile is frozen. We attribute
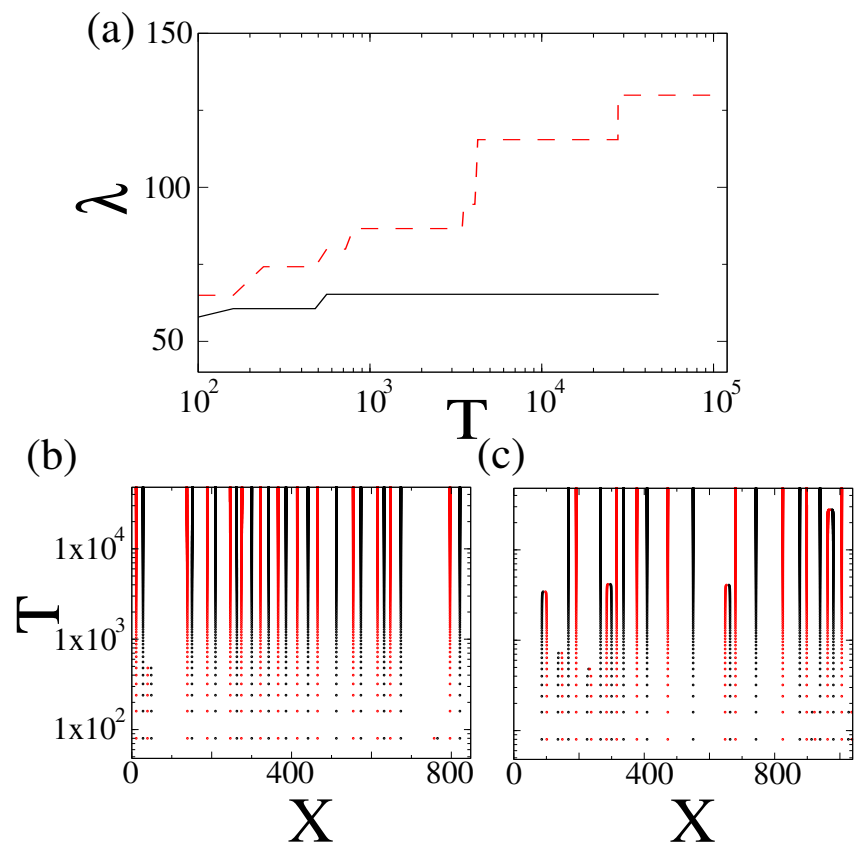

FIG. 2. Non-conserved dynamics. (a) Average wavelength as a function of time. Black solid line corresponds to $\gamma=2$, and the red dashed line to $\gamma=3$. (b) Zeros of the membrane profile for $\gamma=2$, and (c) for $\gamma=3$. Black points correspond to the condition $h=0$ and $\partial_{x} h>0$, and red points correspond to $h=0$ and $\partial_{x} h<0$.

the absence of perpetual coarsening to the existence of oscillatory interactions between the kinks, as already discussed in Refs. [16, 27.

\section{Discussion on tension effects}

In summary, tension leads to a threshold above which coarsening is restored, and in addition, tension can induce changes in the spatial organization of the adhesion patches below the threshold.

Using a substrate between the two gaps $\sim 10$ to $20 \mathrm{~nm}$, with bending rigidity and van der Waals attraction as in Refs. [9, 16, 17, we obtain that the critical tension,

$$
\sigma_{c}=\gamma_{c} \frac{\mathcal{U}_{0}^{1 / 2} \kappa^{1 / 2}}{h_{0}}
$$

is $\sigma_{c} \sim 10^{-2} \mathrm{Jm}^{-2}$. This value is larger than the tensions $\sigma \sim 10^{-5}-10^{-3} \mathrm{~J}_{\mathrm{m}} \mathrm{m}^{-2}$ reported by experiments [17, 32]. Hence, the frozen states should not be eliminated by membrane tension in usual experimental conditions.

\section{ASYMMETRIC POTENTIAL}

Another natural extension of our model is to consider asymmetric adhesion potentials. Such an asymmetry occurs when the membrane is sandwiched between two different substrates, and therefore is expected to be the rule 
(a)

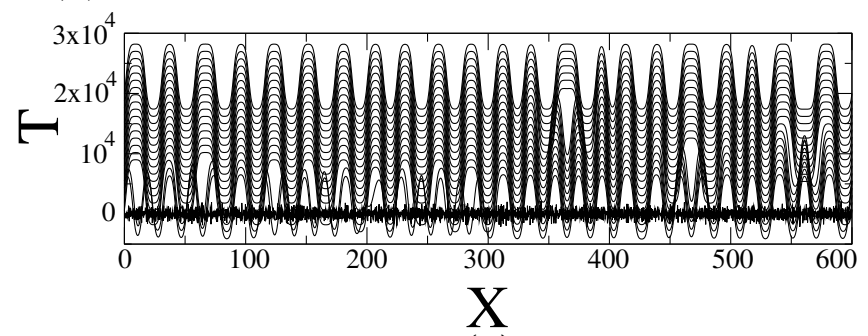

(b)
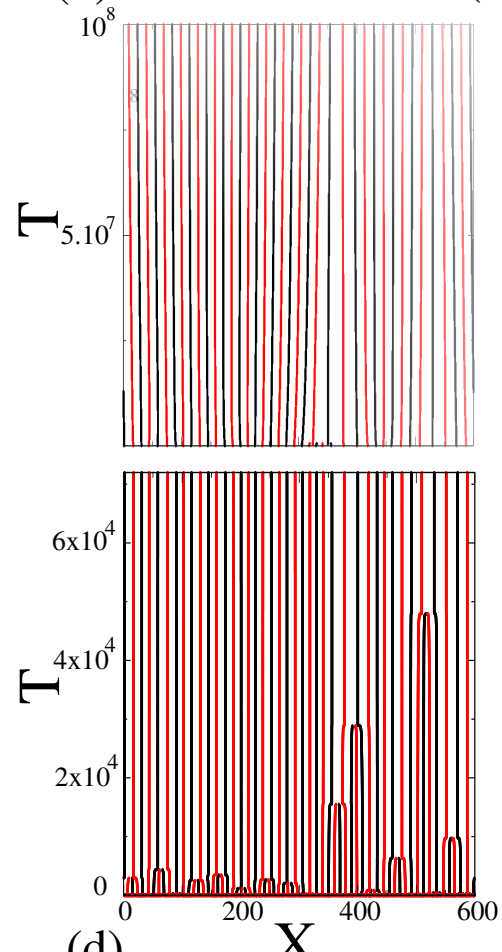

(d)

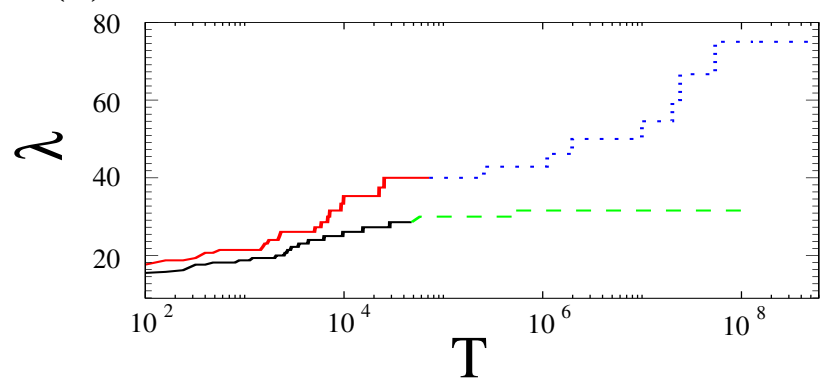

FIG. 3. Conserved dynamics. (a) Membrane profile as a function of time for $\gamma=2$. (b) Trajectories of the zeros of the membrane profile. Bottom: full simulation; top: subsequent dynamics obtained from the kink model. (c) Same plots as (b) for $\gamma=3$. (d) Average wavelength as a function of time. Interrupted coarsening is found in the lower curve with $\gamma=2$ : the black solid line corresponds to the full dynamics, and the green dashed line is obtained from the kink model for $\gamma=2$. Endless coarsening is found in the upper curve with $\gamma=3$ : red solid line for full dynamics and blue dotted line for the kink model.

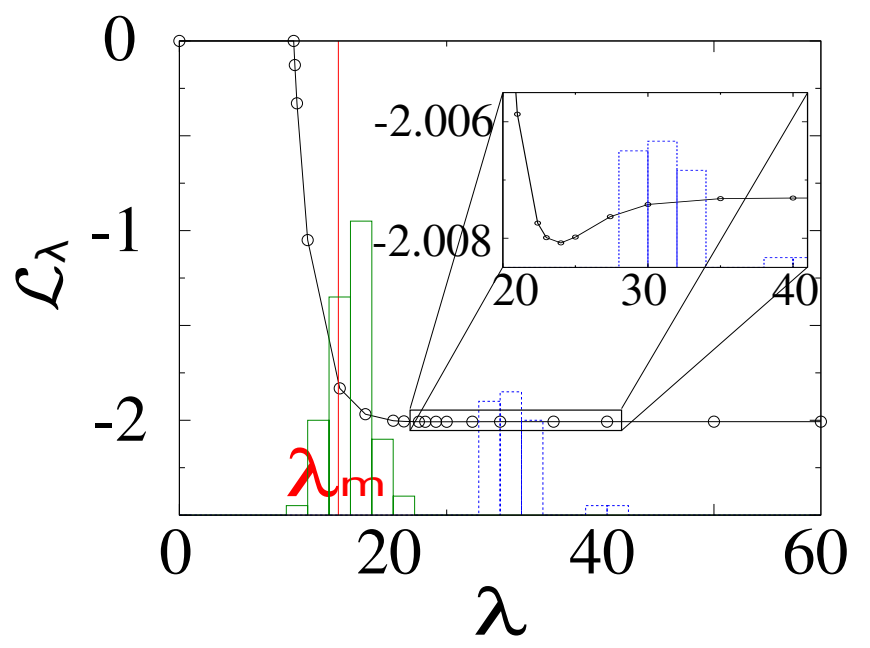

FIG. 4. In black with circles, $\mathcal{L}_{\lambda}$ obtained by simulations as a function of $\lambda$ for $\gamma=2$ and mean height $\bar{H}=0$. The red vertical line gives the position of the most unstable wavelength $\lambda_{m}$ in the linear analysis. The solid green histogram is the distribution of wavelengths for the early dynamics of the membrane with $\gamma=2$, and the dotted blue histogram is the long-time distribution.

rather than the exception. As an example, the adhesion of a cell membrane sandwiched between the cytoskeleton and the extracellular matrix has no reason to be symmetric. Moreover, model systems with vesicles and polymer brushes exhibits a controlled asymmetric double-well potential [10, 11].

\section{A. Asymmetric TDGL4 equation}

In order to control the asymmetry within a simple model, we assume a potential of the form

$$
\mathcal{U}(h)=\mathcal{U}_{0}\left[U_{s}(H)+\beta H\right]
$$

where $U_{s}(-H)=U_{s}(H)$ is symmetric, and $\beta$ is a constant tuning the asymmetry. Within this model, the normalized force acting on the membrane is now:

$$
F_{Z}=-\partial_{X}^{4} H+\gamma \partial_{X X} H-U_{s}^{\prime}(H)-\beta
$$

In the following discussion on the consequences of potential asymmetry, we only consider the tensionless case $\gamma=0$. As seen from Eq. (5) or (8), in the conserved case this force intervenes only via its partial derivative with respect to $x$. As a consequence, the constant term in the force, i.e. the asymmetry, is irrelevant for impermeable walls.

In contrast, the asymmetry plays an important role in the non-conserved case. In order to discuss this case in more details, we use the quartic potential Eq.24 for the symmetric part $U_{s}$, and the normalized membrane evolution equation then reads:

$$
\partial_{T} H=-\partial_{X}^{4} H+H_{m}^{2} H-H^{3}-\beta .
$$


(a)

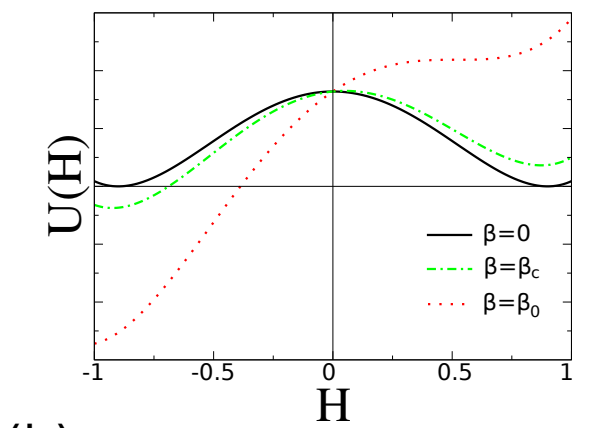

(b)

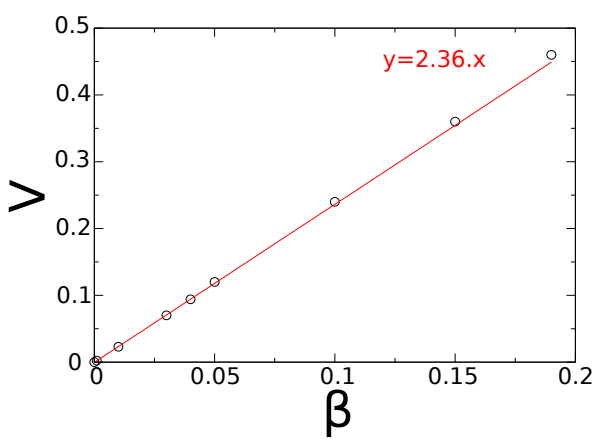

(c)

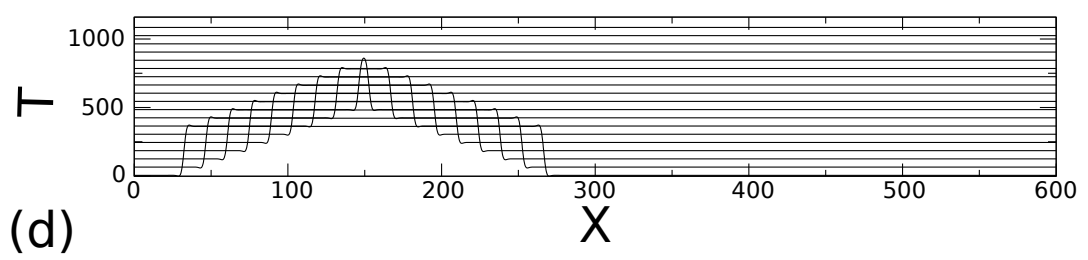

(d)

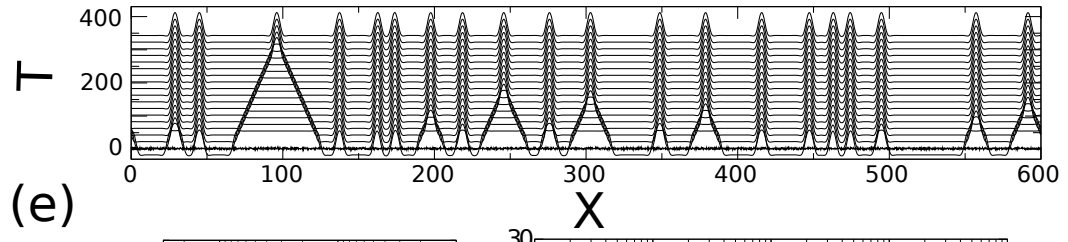

(e)
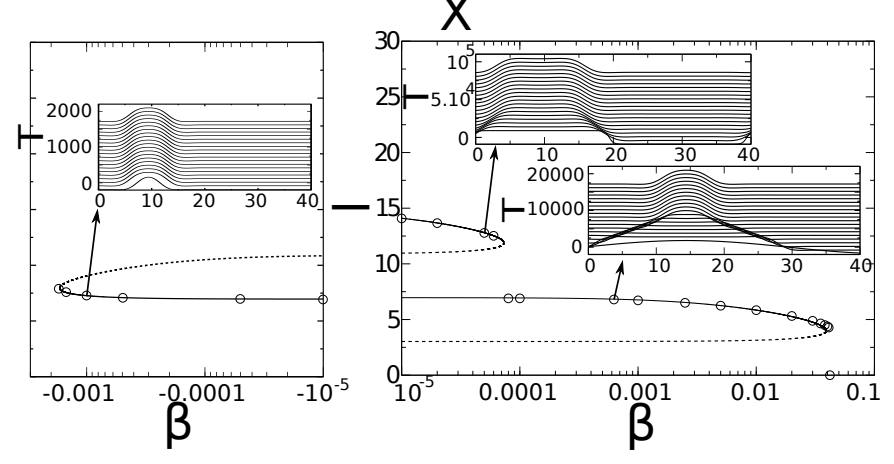

FIG. 5. Membrane dynamics in an asymmetric double-well potential. (a) Asymmetric potential $U(H)$ for $H_{m}=0.9$, and $\beta=\beta_{0} \simeq 0.281$ or $\beta=\beta_{c} \approx 0.041$. (b) Velocity $V$ of an isolated kink as a function of $\beta$. Circles: full simulations. The red solid line is the linear prediction for small $\beta$, see text. (c,d) Non-conserved dynamics in an asymmetric potential for: (c) $\beta=0.1$ -which is intermediate between $\beta_{c}$ and $\beta_{0}$, and (d) $\beta=0.04$-which is smaller than $\beta_{c}$. (e) Steady-state distance length $l$ of an isolated adhesion patch on the upper wall as a function of $\beta$. The circle are found from full simulation, and the the line is the prediction of the kink model. The solid line represents the stable steady-states, and the dashed line the unstable ones. The insets show the dynamics of the membrane in specific cases.

First, note that for very strong asymmetries $|\beta|>\beta_{0}=$ $2 H_{m}^{3} / 3^{3 / 2} \simeq 0.385 H_{m}^{3}$, there is only one minimum, and the dynamics consists in a trivial relaxation to a flat membrane in a single potential well.

For moderate $\beta$, i.e. $\beta_{0}>|\beta|>\beta_{c} \approx 0.056 H_{m}^{3}$, the numerical solution of Eq. $(29)$ indicates that kinks drift at constant velocity and annihilate so that the whole membrane moves to the lowest potential minimum in finite time, as shown in Fig 5(c). For smaller values of the asymmetry $|\beta|<\beta_{c}$, the drift of kinks is still observed, but depending on the initial conditions, the membrane sometimes ends up in a configuration with frozen asymmetric adhesion patches in the unfavorable well of the potential, as shown in Fig.5(d). In the following subsections we discuss and analyze quantitatively these results.

\section{B. Kink drift}

Let us first discuss the kink drift. Consider an isolated kink separating two adhesion domains in the two potential wells. If the depth of the potential wells are different, the total energy can be decreased by a displacement of the kink in the direction that increases the size of the ad- hesion domain with the lowest energy. We assume that a kink drifts at some constant velocity $V$, with a profile $H_{k}(X-V t)$. Multiplying Eq. 29$)$ by $\partial_{X} H_{k}$ and integrating over $x$, we find

$$
V=\beta \frac{B_{0}}{B_{1}}
$$

Due to the change of sign of $B_{0}$, kinks and antikinks drift in opposite directions (due to symmetry, the absolute values of their drift velocities are also equal).

In general, the kink profile $H_{k}$ depends on $\beta$, so that $V$ might exhibit a complex dependence on $\beta$ via $B_{0}$ and $B_{1}$ in the r.h.s. of Eq. (30). However, we expect from Eq. (30) that $V$ should be linear in $\beta$ when $\beta \ll 1$. In addition, due to the $H \rightarrow-H$ symmetry of Eq. (29) at $\beta=0$, both $B_{0}=H_{k}(+\infty)-H_{k}(-\infty)$ and $B_{1}=\int_{-\infty}^{+\infty} \mathrm{d} X\left(\partial_{X} H_{k}\right)^{2}$ depend only on $\beta^{2}$. As a consequence, the first corrections to linearity in the dependence of $V$ in $\beta$ should be cubic, and the linear approximation could be a good approximation up to finite values of $\beta$. This result is confirmed in Fig. 5(b). Furthermore, the prefactor of this linear relation can be calculated from the kink profile at $\beta=0$. Using the numerical evaluation of the static kink profile at $\beta=0$ with 
$H_{m}=0.9$, we find from Eq. 30 $V \approx 2.36 \beta$. This is in good agreement with the direct numerical measurement of the kink drift velocity at small $\beta$ in Fig. 5(b).

\section{Asymmetric frozen adhesion patches}

To perform a systematic analysis of the asymmetric frozen patches, we have implemented the numerical solution of Eq. 29). Depending on the initial condition and on $\beta$, one may obtain different final states. Some examples are shown in Fig. 5 (c,d).

In order to rationalize these results, we have plotted in Fig. 5(e) the size of a single, steady domain in a large system as a function of $\beta$. In this figure, the steadystate branches with $\beta>0$ represent finite-size patches in the higher energy potential well, while the branches with $\beta<0$ represent finite-size adhesion patches in the lower potential well. Each simulation point is obtained from a suitable choice of initial condition. The lower branch for $\beta>0$ is obtained with a sinusoidal initial condition. The upper branch is obtained from an initial condition with a localized domain formed by superposition of tanh functions. Finally, the lower branch with $\beta>0$ was used as an initial condition to obtain the steady-state solutions in the branch with $\beta<0$.

Globally Fig.5(e) suggests the presence of several branches of steady-state solutions for $|\beta|<0.041$, while no steady-state patch is observed for $|\beta|>0.041$.

These results can be described quantitatively within the kink model. Indeed, the kink model Eq. 100 can be simply extended to account for asymmetry, leading to:

$$
\dot{X}_{n}=\frac{1}{B_{1}}\left(\Delta \tilde{R}_{n}+\beta B_{0}\right) .
$$

where the sign of $B_{0}$ alternates between + for kinks and - for antikinks. Considering a single adhesion domain on the upper wall, centered at $x=0$, we have a kink at $X_{1}=-\ell / 2$, and an antikink at $X_{2}=\ell / 2$. We therefore find that steady-states, corresponding to $\partial_{t} \ell=0$, obey

$$
\beta=-\frac{\tilde{R}(\ell)}{\left|B_{0}\right|} .
$$

In addition, from Eq. 19):

$$
\tilde{R}(\ell)=2 A^{2} U_{m}^{\prime \prime} \cos \left[\frac{\ell U_{m}^{\prime 1 / 4}}{2^{1 / 2}}+2 \alpha\right] \exp \left[-\frac{\ell U_{m}^{\prime 1 / 4}}{2^{1 / 2}}\right] .
$$

For $\beta \ll 1$, the constants can be determined numerically from the symmetric case at $\beta=0$, leading to: $\left|B_{0}\right| \approx 2 H_{m}, A=0.87$, and $\alpha=2.72$ [16]. As shown in Fig. 5(e), these assumptions allow one to obtain a good quantitative agreement between the kink model (solid and dashed lines), and the steady-states observed in the simulations (symbols).
Furthermore, the stability of these steady-states can also be understood within the kink model. Indeed, consider a stationary state with two kinks separated by the distance $\ell$. Then, assuming a perturbation of $\ell$ equal to $\delta \ell \propto \exp (i \omega T)$, we obtain from Eq. 31

$$
i \omega=-\frac{2 \tilde{R}^{\prime}(\ell)}{B_{1}}
$$

indicating that if $\tilde{R}^{\prime}(\ell) \leq 0$ the state is unstable. Using Eq. $(32)$, we find that the steady-state is unstable if $\partial_{\ell} \beta \geq 0$. This is in agreement with the results of Fig. 5(e), where no steady-state is observed in the unstable branches with $\partial_{\ell} \beta \geq 0$, indicated with a dashed line.

\section{Discussion on asymmetry}

In summary, in the non-conserved case, a finite potential asymmetry is needed in order to eliminate the frozen adhesion patches. However, we recall that asymmetry in the depth of potential wells has no effect in the presence of impermeable substrates.

Asymmetric two-state adhesion potentials have been obtained in experiments [10, 11] using competition between a medium-range repulsion created by polymer brushes (mimicking the glycocalyx of cells), and a short range attraction resulting from the attachment of ligandreceptor pairs.

It is difficult to provide precise quantitative predictions for these experiments because the potentials of Refs. [10, 11] do not exhibit the simple quartic form with a linear bias that we assumed here in Eqs. 24 27]. However, asymmetries can be compared using a simple dimensionless parameter

$$
b=\frac{U\left(H_{m+}\right)-U\left(H_{m-}\right)}{U\left(H_{M}\right)-\left[U\left(H_{m+}\right)+U\left(H_{m-}\right)\right] / 2},
$$

where $H_{m \pm}$ are the positions of the two minima of the potential, and $H_{M}$ is the position of the maximum. Our model potential Eqs.2427) leads to $b \approx 8 \beta / H_{m}^{3}$ for small $\beta$. As a consequence, the critical asymmetry above which patches cannot survive is $b_{c} \approx 8 \beta_{c} / H_{m}^{3} \approx 0.45$.

We find a smaller asymmetry $b \approx 0.3$ from Fig. 5 of Ref. [10, and a larger asymmetry $b \approx 1.5$ for Ref. [11, suggesting that adhesion patches may survive the asymmetry for the potential of Ref. [10, but not for that of Ref. [11. This conclusion should not be taken as a quantitative statement. However, it suggests that both situations could be observable in this type of experiments, provided that the substrate is permeable.

\section{THERMAL NOISE}

Another important physical ingredient which is able to affect the frozen states and restore coarsening is thermal 
fluctuations. Lipid membranes usually exhibit a bending rigidity of the order of $30 k_{B} T$, and are therefore subject to significant thermal fluctuations. In this section, we investigate the consequences of thermal fluctuations on membrane dynamics.

\section{A. Noisy kink dynamics}

We include Langevin forces in the kink dynamics equations following the same lines as in Ref. [30, 31. As discussed in Appendix A, we then have for the nonconserved and conserved cases respectively

$$
\begin{aligned}
\dot{X}_{n}= & \frac{1}{B_{1}} \Delta \tilde{R}_{n}+\zeta_{n}(T), \\
\dot{X}_{n}= & \frac{1}{B_{0}^{2}}\left(\frac{\tilde{R}_{n+3 / 2}-\tilde{R}_{n-1 / 2}}{\ell_{n+1 / 2}}+\frac{\tilde{R}_{n+1 / 2}-\tilde{R}_{n-3 / 2}}{\ell_{n-1 / 2}}\right) \\
& +\frac{\xi_{n+1 / 2}(T)}{\ell_{n+1 / 2}^{1 / 2}}+\frac{\xi_{n-1 / 2}(T)}{\ell_{n-1 / 2}^{1 / 2}},
\end{aligned}
$$

where the Langevin forces $\zeta$ and $\xi$ are zero-average white Gaussian noise. Their correlations read

$$
\begin{aligned}
\left\langle\zeta_{n_{1}}\left(T_{1}\right) \zeta_{n_{2}}\left(T_{2}\right)\right\rangle & =2 D_{\zeta} \delta_{n_{1} n_{2}} \delta\left(T_{1}-T_{2}\right) \\
\left\langle\xi_{n_{1}}\left(T_{1}\right) \xi_{n_{2}}\left(T_{2}\right)\right\rangle & =2 D_{\xi} \delta_{n_{1} n_{2}} \delta\left(T_{1}-T_{2}\right)
\end{aligned}
$$

where $\delta_{n_{1} n_{2}}$ and $\delta(t)$ are respectively the Kronecker symbol and Dirac delta function. In the conserved case Eq.(37), we have neglected the subdominant terms proportional to $B_{2}$ in Eq. (11), which are not expected to affect qualitatively the asymptotic dynamics.

The noise amplitudes are derived in Appendix A using the fluctuation-dissipation theorem:

$$
\begin{aligned}
& D_{\zeta}=\frac{k_{B} T}{B_{1} \mathcal{U}_{0}^{3 / 4} h_{0}^{1 / 2} \kappa^{1 / 4}} \\
& D_{\xi}=\frac{k_{B} T}{B_{0}^{2} \mathcal{U}_{0}^{3 / 4} h_{0}^{1 / 2} \kappa^{1 / 4}}
\end{aligned}
$$

We have implemented numerically these Langevin equations. The details of the numerical scheme are described in Appendix B

\section{B. Activated coarsening}

The numerical solution of Eqs.(36 37) indicates that thermal fluctuations always lead to coarsening at long times. As shown in Fig. 6(a,b), the coarsening exponent $1 / 2$ for noisy-TDGL4 and $1 / 3$ for noisy-CH4, are the same as those found for noisy-TDGL and noisy- $\mathrm{CH}$ respectively. The same exponents would be observed if the deterministic terms in r.h.s. of Eqs. (36 37) were absent. This suggests that the precise form of the linear terms (second or fourth order) is irrelevant at long times, and asymptotic coarsening is controlled only by the noise and the conservation law.

In contrast, the short-time behavior is strongly influenced by the deterministic stabilizing terms. In the TDGL and CH cases, the coarsening is logarithmic in the early time dynamics, as in the deterministic case [30, 31. Then, it crosses over to an asymptotic power-law in the late stages. This well known behavior is shown in Fig. 6(a). Similarly, the TDGL4 and CH4 noisy kink dynamics behave like the deterministic dynamics at short times, i.e. with arrested dynamics. Then, we find a crossover to the expected power-law behavior, as seen in Fig. 6(b).

The crossover time $t_{c}$ to the coarsening regime in TDGL4 and CH4 exhibits an exponential dependence in the noise amplitude:

$$
\begin{aligned}
t_{c} & =t_{c 0} \exp \left[\frac{E_{0}}{\hat{D}}\right] \\
& =t_{c 0} \exp \left[E_{0} 2^{1 / 2} A^{2} U_{m}^{\prime \prime} \frac{3 / 4}{\mathcal{U}_{0}^{3 / 4} h_{0}^{1 / 2} \kappa^{1 / 4}}\right. \\
k_{B} T &
\end{aligned}
$$

where we have used the normalized noise amplitude

$$
\hat{D}=\frac{k_{B} T}{2^{1 / 2} A^{2} U_{m}^{\prime \prime}{ }^{3 / 4} \mathcal{U}_{0}^{3 / 4} h_{0}^{1 / 2} \kappa^{1 / 4}},
$$

defined in the normalized equations of Appendix B.

Note that, in order to obtain the dependence of our results on the kink parameters $\left(A, U_{m}^{\prime \prime}, B_{0}\right.$, and $\left.B_{1}\right)$, we have performed the noisy kink simulations with a special set of normalized coordinates defined in Appendix B. This dependence in the kink parameters then appears explicitly when going back to the coordinates used in main text.

We have measured $t_{c}$ using an arbitrary threshold wavelength $\lambda_{c}$ from the relation $\lambda\left(t_{c}\right)=\lambda_{c}$, where $\lambda(t)$ is the average distance between kinks at time $t$. We started from randomly distributed kinks with an initial average separation $\lambda(t=0)=2.30 \times U_{m}^{\prime \prime-1 / 4}$ corresponding to the most unstable wavelength within the kink model. We obtain $E_{0} \approx 0.095$ for TDGL4 with $\lambda_{c}=17.0 \times U_{m}^{\prime \prime-1 / 4}$, and $E_{0} \approx 0.085$ for $\mathrm{CH} 4$ with $\lambda_{c}=7.92 \times U_{m}^{\prime \prime-1 / 4}$.

This thermal activation of coarsening can be intuitively understood from the need to overcome small energy barriers corresponding to the oscillatory interactions between kinks.

The existence of barriers can be quantitatively discussed within the kink approximation. One basic assumption underlying the kink description, and explored in details in Ref. [27], is that the profile between two kinks can be approximated by that of a periodic steady-state. Using this approximation, we may design an expression 

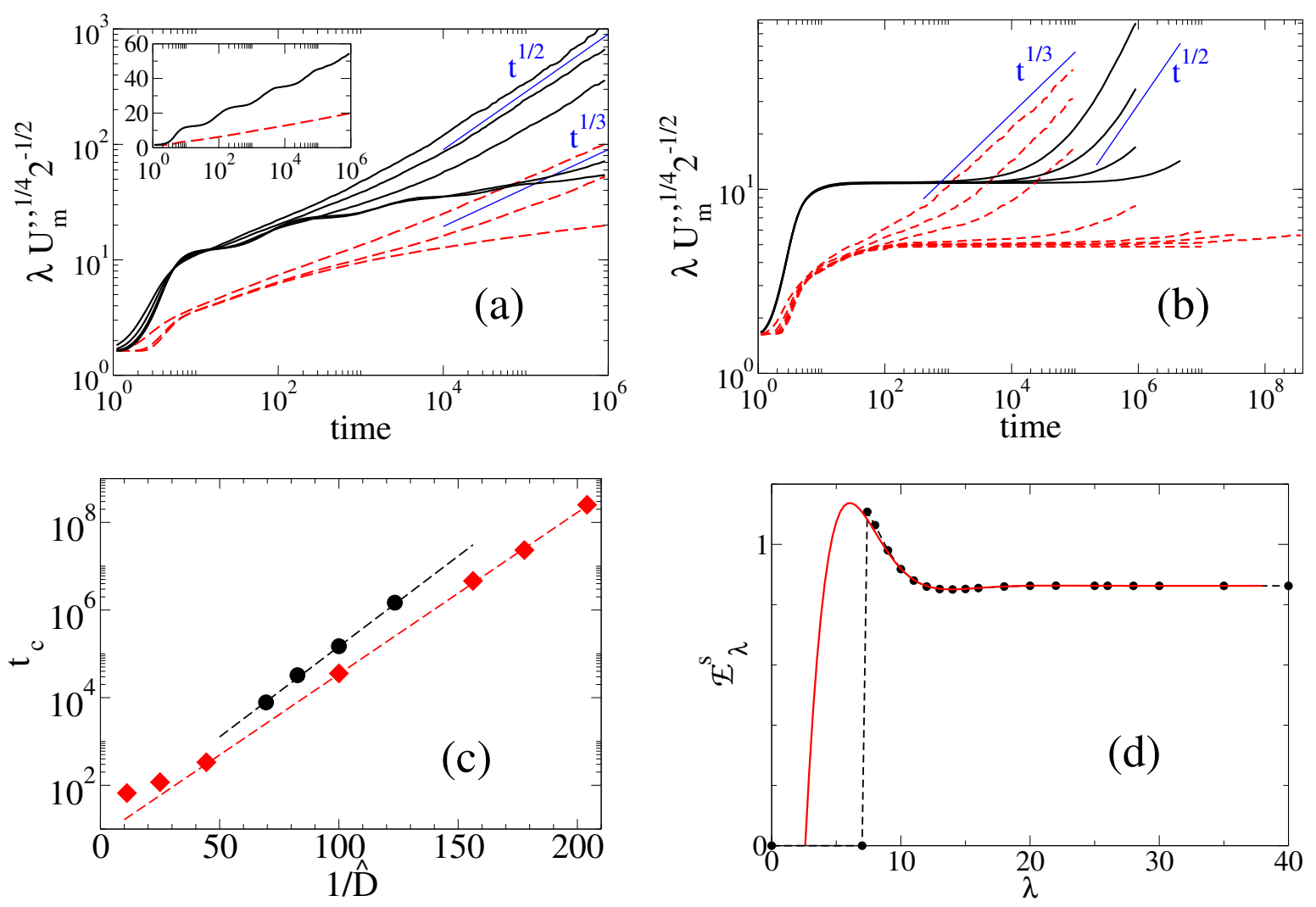

FIG. 6. Activated coarsening. (a) Normalized average distance $\lambda U_{m}^{1 / 4} 2^{-1 / 2}$ between kinks as a function of normalized time. Noisy-TDGL kink dynamics: black solid lines, with from bottom to top $\hat{D}=0.002,0.01,0.1,0.2,0.3$. Noisy-CH kink dynamics: red dashed lines, with from bottom to top $\hat{D}=0.002,0.1,0.3$. Thin lines are expected power-laws at long times, and the inset in semi-log coordinates shows logarithmic coarsening in the case of low noise amplitude $\hat{D}=0.002$ for TDGL (solid line), and CH (dashed line). (b) Normalized average distance $\lambda U_{m}^{1 / 4} 2^{-1 / 2}$ between kinks as a function of normalized time. Noisy-TDGL4 kink dynamics: black solid lines, with from bottom to top $\hat{D}=0.09,0.1,0.11,0.12$. Noisy-CH4 kink dynamics: red dashed lines, with from bottom to top $\hat{D}=0.07,0.070 .075,0.08,0.10 .15,0.20 .3$. (c) Time $t_{c}$ for the initiation of coarsening: discs for TDGL4, and diamonds for CH4. (d) Energy of a periodic steady-state $\mathcal{E}_{\lambda}^{s}$ as a function of its period $\lambda$ for the potential defined in Eq. 24), with $H_{m}=0.9$. The symbols (dashed line) indicate the full numerical solution of the membrane profile. The red solid line is the large $\lambda$ approximation from Eq. 444.

for the energy of a periodic steady-state with a large $\lambda$

$$
\begin{aligned}
& \mathcal{E}_{\lambda}^{s}=2 \mathcal{E}_{\text {kink }} \\
+ & 4 A^{2} U_{m}^{\prime \prime 3 / 4} \sin \left[\frac{\lambda U_{m}^{\prime \prime 1 / 4}}{2^{3 / 2}}+2 \alpha-\frac{\pi}{4}\right] \exp \left(\frac{-\lambda U_{m}^{\prime \prime 1 / 4}}{2^{3 / 2}}\right)
\end{aligned}
$$

where $\mathcal{E}_{\text {kink }}$ is the energy of an isolated kink. The detailed derivation of this result is reported in Appendix $\mathrm{A}$. A comparison to the exact energy computed numerically in Fig. 6.(d) shows that Eq. (44) is a good approximation for the energy for large $\lambda$. When $\lambda<8.35 . . \times U_{m}^{-1 / 4}$, the full numerical solution appears to be unstable. The expression (44) exhibits a maximum around $\lambda \approx 6.83 . . \times$ $U_{m}^{-1 / 4}$, and for smaller distances, pairs of kinks are expected to experience an attraction leading to annihilation. The energy barrier, i.e. the difference between the minimum energy and the maximum energy that can be reached before annihilation is similar in both cases. Since we wish to approximate the profile between two kinks by half a periodic steady-state, the effective energy barrier is half the barrier observed in Fig. 6(d). We find similar values $E_{b}^{t h} \approx 0.143$ from Eq. (44), and $E_{b}^{\text {num }} \approx 0.13$ from the numerical solution of the full profile. Hence the kink model provides a reasonable description of the energy barrier.

Using Eq. 42 with Eqs. 40[41, we find the expected value of $E_{0}$ for both the conserved and non-conserved cases:

$$
E_{0}=\frac{E_{b}}{2^{1 / 2} A^{2} U_{m}^{\prime \prime 3 / 4}}
$$

Using the above-mentioned value $E_{b}^{t h} \approx 0.143$, we finally obtain $E_{0} \approx 0.093$, in good agreement with the values extracted from the exponential dependence of $t_{c}$ (see above). Hence, Eq. (44) provides a quantitative understanding of the origin of the energy barriers controlling the activation of coarsening in noisy kink dynamics. 


\section{Discussion on noise}

The results of this section can be interpreted qualitatively within a simple picture where coarsening is controlled by the competition between two timescales. The first timescale $t_{\text {diff }} \sim \lambda^{\theta}$ is the time needed for noninteracting kinks moved only by Langevin forces to collide with their neighbors, which are initially located at a typical distance $\lambda$. From the dimensional analysis of Eqs. 36.37$)$, one finds $\theta=2$ for the non-conserved case, and $\theta=3$ for the conserved case.

In tension-dominated models, i.e. TDGL or $\mathrm{CH}$, the second time-scale is the time $t_{\text {int }}$ needed for two neighboring kinks to annihilate due to their deterministic mutual attraction. Since this attraction decreases exponentially with the distance, we have $t_{\text {int }} \sim \mathrm{e}^{\lambda}$. Here, kink random motion and deterministic interactions act in parallel, and the shortest of the two timescales dominates. Thus, initially for small $\lambda$, and if the noise strength is small enough, one has $t_{\text {int }} \ll t_{\text {diff }}$, leading to a dynamical behavior dominated by $t_{\text {int }}$, with the standard logarithmic coarsening law $\lambda \sim \ln t$. However, at large times $t_{\text {int }} \gg t_{\text {diff }}$. As a consequence, the dynamics is dominated by the random motion of the kinks, and one finds the power-law behavior $\lambda \sim t^{\theta}$.

In bending-dominated models, i.e. TDGL4 or CH4, the second timescale is the time $t_{c}$ (given in Eq.42) for a pair of kinks to overcome the energy barrier for collision via thermal fluctuations. Here, kink random motion and the passage over the energy barrier act in series, so that the largest of the two timescales $t_{\text {diff }}$ and $t_{c}$ dominates. Thus, at short times and for small enough noise strength, one has $t_{\text {diff }} \ll t_{c}$, and coarsening is absent. In contrast, at long times $t_{\text {diff }} \gg t_{c}$, and the power-law coarsening with $\lambda \sim t^{\theta}$ is recovered.

As an important remark, the predictions of the previous sections on tension and asymmetry depend on the lengthscales $h_{0}$ and $\left(\kappa / \mathcal{U}_{0}\right)^{1 / 2}$, and the timescales $h_{0}^{2} \nu^{-1} \mathcal{U}_{0}^{-1}$ or $\mu \kappa^{1 / 2} \mathcal{U}_{0}^{-3 / 2}$ for the non-conserved and conserved cases respectively. These spatial and temporal scales naturally extend to two-dimensional membranes in three-dimensional liquids keeping the same formula and replacing the physical constants $\kappa$ and $\mathcal{U}_{0}$ by the two-dimensional ones which have different dimensions. Hence, we expect the above-mentioned results to catch some of the physical behavior of two-dimensional membranes.

However, in the presence of thermal fluctuations, we now have a relevant energy scale, which is the energy barrier $\mathcal{E}_{b}$. In physical coordinates, it reads

$$
\mathcal{E}_{b}=\mathcal{U}_{0}^{3 / 4} h_{0}^{1 / 2} \kappa^{1 / 4} E_{b}
$$

This energy scale cannot be naturally extended to two dimensional membranes, and a simple substitution of the energy parameters $\kappa$ and $\mathcal{U}_{0}$ by their two-dimensional counterparts provides an expression of $\mathcal{E}_{b}$ which does not have the dimension of an energy. Physically, con- fined two-dimensional membranes would exhibit onedimensional domain walls instead of kinks. The collision and annihilation of two one-dimensional domain walls should occur locally in a region whose spatial extent should be fixed by the physics of the two-dimensional problem. It is therefore clear that we cannot directly use the result of our model to perform quantitative predictions about two-dimensional membranes. However, there should still be an energy barrier for domain wall collision in two-dimensional membranes.

\section{CONCLUSION}

In summary, we have shown that the frozen patches observed in the 1D dynamics of membranes with bending rigidity survive up to a finite threshold to various other physical driving forces such as tension, potential asymmetry, and thermal fluctuations. Beyond these thresholds, coarsening is restored. However the transition to coarsening exhibits different scenarios in these three cases.

(i) In the presence of tension, there is a critical tension $\sigma_{c}$ above which the oscillations of the membrane profile disappear, leading to monotonic attractive interactions similar to that of the standard TDGL or $\mathrm{CH}$ equations. The orders of magnitude indicate that the tensions usually observed in experiments are smaller than the critical tension $\sigma_{c}$, showing that frozen adhesion patches should still exist in typical experimental conditions.

(ii) An asymmetry in the depth of the two potential wells has no effect on the conserved case, where walls are impermeable. However, in the non-conserved case, i.e. for permeable walls, kinks and antikinks experience drift forces in opposite directions, which are able to overcome the oscillatory kink-kink interactions beyond some finite threshold. The critical asymmetry above which frozen patches cannot be observed is comparable to the asymmetry obtained in experimental works [10,11, suggesting that an asymmetry-induced transition could be observed experimentally in the presence of impermeable walls.

(iii) The presence of thermal fluctuations always lead to coarsening at long times. Nevertheless, the time required for the system to undergo coarsening depends exponentially on the noise strength, i.e. on the temperature. Hence, for temperatures smaller that the typical energy barrier for collision and annihilation, the coarsening process cannot be observed. Although we expect energy barriers to exist for fully two-dimensional membrane, we cannot conclude on the quantitative value of the barriers within our model with a one-dimensional membrane.

Additional differences could appear between onedimensional and two-dimensional membranes. For example, kinks in one-dimensional membranes mimic flat domain walls separating adhesion zones between the upper and the lower walls. However, it is clear that any effect related to the curvature of these domain walls cannot be accounted for within our model with one-dimensional 
membranes.

To conclude, our study of idealized zero-thickness, and one-dimensional interfaces with bending rigidity sandwiched between two flat walls, aimed at capturing qualitatively some of the complex dynamics of lipid membranes in confined biological environments. Our results show that bending rigidity is at the origin of a unique zoology of dynamical behaviors, with finite-size patches that are robust to various physical perturbations up to a finite threshold.

\section{ACKNOWLEDGMENTS}

We acknowledge support from the Agence Nationale de la Recherche Biolub Grant (ANR-12-BS04-0008).

\section{Appendix A: Derivation of the noise amplitude in the kink model for thermal noise}

Here, we relate kink dynamics to an energetic picture. The amplitude of thermal noise then follows directly from this derivation using the fluctuation-dissipation theorem.

\section{Energy and force acting on a kink}

We start by decomposing the membrane into regions. The region $n+1 / 2$ is located betweens kinks $n$ and $n+1$. The total energy

$$
\mathcal{E}=\int d x\left[\frac{\sigma}{2}\left(\partial_{x} h\right)^{2}+\frac{\kappa}{2}\left(\partial_{x x} h\right)^{2}+U(h)\right],
$$

is then equal to the sum of the corresponding energy contributions $\mathcal{E}_{n+1 / 2}$ :

$$
\mathcal{E}=\sum_{n} \mathcal{E}_{n+1 / 2}
$$

In the kink picture, the energy $\mathcal{E}_{n+1 / 2}$ is approximated by half the energy $\mathcal{E}_{\lambda}^{s}$ of a periodic steady-state of wavelength $\lambda=2 l_{n+1 / 2}$, where $l_{n+1 / 2}=x_{n+1}-x_{n}$. This reads

$$
\mathcal{E}_{n+1 / 2}=\left.\frac{1}{2} \mathcal{E}_{\lambda}^{s}\right|_{\lambda=2 l_{n+1 / 2}} .
$$

The energy $\mathcal{E}_{n+1 / 2}$ then only depends on the positions of the neighboring kinks at $x_{n}$ and $x_{n+1}$, the force experienced upon the motion of the $n$-th kink is

$$
\begin{aligned}
F_{n} & =-\frac{d}{d x_{n}} \mathcal{E} \\
& =\partial_{l_{n+1 / 2}} \mathcal{E}_{n+1 / 2}-\partial_{l_{n-1 / 2}} \mathcal{E}_{n-1 / 2} \\
& =\left.\partial_{\lambda} \mathcal{E}_{\lambda}^{s}\right|_{\lambda=2 l_{n+1 / 2}}-\left.\partial_{\lambda} \mathcal{E}_{\lambda}^{s}\right|_{\lambda=2 l_{n-1 / 2}} .
\end{aligned}
$$

In addition, from the expression

$$
\mathcal{E}_{\lambda}^{s}=\int_{0}^{\lambda} d x\left[\frac{\sigma}{2}\left(\partial_{x} h\right)^{2}+\frac{\kappa}{2}\left(\partial_{x x} h\right)^{2}+U(h)\right],
$$

we find

$$
\partial_{\lambda} \mathcal{E}_{\lambda}^{s}=\frac{1}{\lambda} \int_{0}^{\lambda} d x\left[-\frac{\sigma}{2}\left(\partial_{x} h\right)^{2}-\frac{3 \kappa}{2}\left(\partial_{x x} h\right)^{2}+U(h)\right] .
$$

Moreover, one can easily check that

$\partial_{x} h \frac{\delta E}{\delta h}=\partial_{x}\left[\frac{\sigma}{2}\left(\partial_{x} h\right)^{2}-\kappa \partial_{x} h \partial_{x x x} h+\frac{\kappa}{2}\left(\partial_{x x} h\right)^{2}-U(h)\right]$.

A periodic steady-state $h^{s}(x)$ by definition obeys $\delta E / \delta h=0$, and

$$
-\frac{\sigma}{2}\left(\partial_{x} h^{s}\right)^{2}+\kappa \partial_{x} h \partial_{x x x} h^{s}-\frac{\kappa}{2}\left(\partial_{x x} h^{s}\right)^{2}+U\left(h^{s}\right)=U^{*}
$$

where $U^{*}$ is a constant. As a consequence, Eq. A6 can be rewritten as

$$
\partial_{\lambda} \mathcal{E}_{\lambda}^{s}=U^{*}
$$

The relation $\mathrm{A} 9$ can be used to express the force of the $n$th kink as

$$
F_{n}=\left.U_{\lambda}^{*}\right|_{\lambda=2 l_{n+1 / 2}}-\left.U_{\lambda}^{*}\right|_{\lambda=2 l_{n-1 / 2}} .
$$

Since Eq. A8 is valid everywhere in a steady-state, we can evaluate it in the zone far away from kinks, where

$$
\begin{aligned}
h \approx h_{0} & \left\{H_{M}+R\left[\left(x-x_{n}\right)\left(\mathcal{U}_{0} / \kappa h_{0}^{2}\right)^{1 / 4}\right]\right. \\
& \left.+R\left[\left(x_{n+1}-x\right)\left(\mathcal{U}_{0} / \kappa h_{0}^{2}\right)^{1 / 4}\right]\right\},
\end{aligned}
$$

leading to

$$
\left.U_{\lambda}^{*}\right|_{\lambda=2 l_{n+1 / 2}}=\mathcal{U}_{0} \tilde{R}\left[l_{n+1 / 2}\left(\mathcal{U}_{0} / \kappa h_{0}^{2}\right)^{1 / 4}\right] .
$$

where $\tilde{R}$ is defined in Eq. 33 .

Combining Eq. A12 with Eq. A9, one can compute a convenient expression of the energy of a periodic steadystate:

$$
\mathcal{E}_{\lambda}=2 \mathcal{E}_{\text {kink }}-\mathcal{U}_{0} \int_{\lambda}^{\infty} d x \tilde{R}\left[\frac{\lambda}{2} \frac{\mathcal{U}_{0}^{1 / 4}}{\kappa^{1 / 4} h_{0}^{1 / 2}}\right] .
$$

which leads to Eq.44.

\section{Non-conserved dynamics}

Assuming a simple constant kink mobility $\eta$ (local in space and with no memory effect), we write that the kink velocity is proportional to the force plus a noise term

$$
\dot{x}_{n}=\eta F_{n}+\bar{\zeta}_{n}=\eta \mathcal{U}_{0} \Delta \tilde{R}_{n}+\bar{\zeta}_{n} .
$$

Here, $\bar{\zeta}_{n}$ is a white noise obeying

$$
\left\langle\bar{\zeta}_{n_{1}}\left(t_{1}\right) \bar{\zeta}_{n_{2}}\left(t_{2}\right)\right\rangle=2 \mathcal{D}_{\bar{\zeta}} \delta_{n_{1} n_{2}} \delta\left(t_{1}-t_{2}\right)
$$

where $\mathcal{D}_{\bar{\zeta}}$ is a constant. 
Comparing the deterministic part of Eq. A14 with Eq. 36. , one finds

$$
\eta=\frac{\nu \kappa^{1 / 4}}{2 B_{1} \mathcal{U}_{0}^{1 / 4} h_{0}^{3 / 2}}
$$

We may then use the fluctuation-dissipation theorem, here in the form of an Einstein relation, leading to

$$
\mathcal{D}_{\bar{\zeta}}=\eta k_{B} T=\frac{\nu \kappa^{1 / 4}}{2 B_{1} \mathcal{U}_{0}^{1 / 4} h_{0}^{3 / 2}} k_{B} T .
$$

Finally, the normalized noise $\zeta$ used in Eq. (36) of the main text is related to the noise $\bar{\zeta}$ in physical variables via the relation

$$
\bar{\zeta}_{n}=\frac{\nu \kappa^{1 / 4} \mathcal{U}_{0}^{3 / 4}}{2 h_{0}^{3 / 2}} \zeta_{n}
$$

\section{Conserved dynamics}

In the conserved case, one starts with the observation that, due to mass conservation, the elementary event is the translation of a whole domain instead of that of a single kink. The total force relative to the translation of the domain $n+1 / 2$ is the sum $F_{n+1}+F_{n}$ of the forces acting on the two kinks $n$ and $n+1$. This translational motion is physically realized by a flux $j_{n+1 / 2}$ of liquid under the membrane, to which one associates a mobility $\mu_{n+1 / 2}$ and a noise $\psi_{n+1}$. The flux $j_{n+1 / 2}$ produces a contribution to the motion of the domain $n+1 / 2$ with the velocity $j_{n+1 / 2} /\left|B_{0}\right|$. Hence

$$
\frac{j_{n+1 / 2}}{\left|B_{0}\right|}=\mu_{n+1 / 2}\left(F_{n+1}+F_{n}\right)+\psi_{n+1 / 2},
$$

with the noise correlation

$$
\left\langle\psi_{n_{1}}\left(t_{1}\right) \psi_{n_{2}}\left(t_{2}\right)\right\rangle=2 \mathcal{D}_{\psi, n_{1}} \delta_{n_{1} n_{2}} \delta\left(t_{1}-t_{2}\right),
$$

where $n_{1}$ and $n_{2}$ are half-integers, and $\mathcal{D}_{\psi, n_{1}}$ depends on $n_{1}$ but not on $t$.

Mass conservation then allows one to obtain the kink velocity from the fluxes

$$
\dot{x}_{n}=\frac{j_{n+1 / 2}+j_{n-1 / 2}}{\left|B_{0}\right|},
$$

leading to Eq. (37), with the identification

$$
\mu_{n+1 / 2}=\frac{h_{0}^{1 / 2} \mathcal{U}_{0}^{1 / 4}}{24 \mu B_{0}^{2} \kappa^{1 / 4} \ell_{n+1 / 2}}=\frac{h_{0}}{24 \mu B_{0}^{2} l_{n+1 / 2}}
$$

where we use $l_{n+1 / 2}$ for physical lengths and $\ell_{n+1 / 2}$ for normalized lengths.

Hence, from the fluctuation-dissipation theorem

$$
\mathcal{D}_{\psi, n+1 / 2}=\mu_{n+1 / 2} k_{B} T=\frac{h_{0}}{24 \mu B_{0}^{2} l_{n+1 / 2}} k_{B} T .(\mathrm{A} 23)
$$

Then, defining $\bar{\xi}_{n+1 / 2}=l_{n+1 / 2}^{1 / 2} \psi_{n+1 / 2}$ with the correlations

$$
\left\langle\bar{\xi}_{n_{1}}\left(t_{1}\right) \bar{\xi}_{n_{2}}\left(t_{2}\right)\right\rangle=2 \mathcal{D}_{\bar{\xi}} \delta_{n_{1} n_{2}} \delta\left(t_{1}-t_{2}\right),
$$

we obtain a constant noise amplitude:

$$
\mathcal{D}_{\bar{\xi}}=l_{n+1 / 2} \mathcal{D}_{\psi, n+1 / 2}=\frac{h_{0}}{24 \mu B_{0}^{2}} k_{B} T .
$$

The normalized noise $\xi$ used in Eq. (37) of the main text is related to the noise $\bar{\xi}$ in physical variables via

$$
\bar{\xi}_{n+1 / 2}=\frac{h_{0}^{3 / 4} \mathcal{U}_{0}^{9 / 8}}{24 \mu \kappa^{1 / 8}} \xi_{n+1 / 2}
$$

\section{Appendix B: Numerical schemes for the implementation of noisy kink dynamics}

For the kink simulations with noise, we have further normalized all variables in order to have all numerical prefactors in the kink equations equal to one. For any variable $A$, we associate a normalized simulation variable $\hat{A}$. We have therefore defined the spatial coordinate $\hat{X}=\left(U_{m}^{\prime \prime 1 / 4} / 2^{1 / 2}\right) X$, the time coordinate $\hat{T}=\left(2^{1 / 2} A^{2} U_{m}^{\prime \prime}{ }^{5 / 4} / B_{1}\right) T$, and the noise amplitude $\hat{D}=B_{1} D_{\zeta} /\left(2^{1 / 2} A U_{m}^{\prime \prime}{ }^{3 / 4}\right)$ for the non-conserved case. In the conserved case we use the same spatial coordinate, but different normalizations for time and noise amplitude: the time coordinate is $\hat{T}=A^{2} U_{m}^{\prime \prime}{ }^{3 / 2} /\left(B_{0}^{2}\right) T$, and the noise amplitude is $\hat{D}=B_{0}^{2} D_{\xi} /\left(2^{1 / 2} A U_{m}^{\prime \prime 3 / 4}\right)$. Using these coordinates, the kink model equation read

$$
\begin{aligned}
\dot{\hat{X}}_{n}= & \Delta \hat{R}_{n}+\hat{\zeta}_{n}(T), \\
\dot{\hat{X}}_{n}= & \left(\frac{\hat{R}_{n+3 / 2}-\hat{R}_{n-1 / 2}}{\hat{\ell}_{n+1 / 2}}+\frac{\hat{R}_{n+1 / 2}-\hat{R}_{n-3 / 2}}{\hat{\ell}_{n-1 / 2}}\right) \\
& +\frac{\hat{\xi}_{n+1 / 2}(T)}{\hat{\ell}_{n+1 / 2}^{1 / 2}}+\frac{\hat{\xi}_{n-1 / 2}(T)}{\hat{\ell}_{n-1 / 2}^{1 / 2}},
\end{aligned}
$$

where

$$
\hat{R}(\hat{\ell})=\cos (\hat{\ell}+2 \alpha) \exp (-\hat{\ell}) .
$$

and the Langevin forces $\hat{\zeta}$ and $\hat{\xi}$ are zero-average white Gaussian noise. Their correlations read

$$
\begin{gathered}
\left\langle\hat{\zeta}_{n_{1}}\left(\hat{T}_{1}\right) \hat{\zeta}_{n_{2}}\left(\hat{T}_{2}\right)\right\rangle=\hat{D} \delta_{n_{1} n_{2}} \delta\left(\hat{T}_{1}-\hat{T}_{2}\right), \\
\left\langle\hat{\xi}_{n_{1}}\left(\hat{T}_{1}\right) \hat{\xi}_{n_{2}}\left(\hat{T}_{2}\right)\right\rangle=\hat{D} \delta_{n_{1} n_{2}} \delta\left(\hat{T}_{1}-\hat{T}_{2}\right) .
\end{gathered}
$$

Equations (B1) and (B2) are re-written as evolution equations for the interkink distances $\hat{\ell}_{n+1 / 2}=\hat{X}_{n+1}-$ $\hat{X}_{n}$. The resulting equations have the form

$$
\begin{aligned}
& \dot{\hat{\ell}}_{n+1 / 2}=u_{n}+\hat{\zeta}_{n+1}-\hat{\zeta}_{n} \\
& \dot{\hat{\ell}}_{n+1 / 2}=v_{n}+\frac{\hat{\xi}_{n+3 / 2}}{\hat{\ell}_{n+3 / 2}^{1 / 2}}+\frac{\hat{\xi}_{n+1 / 2}}{\hat{\ell}_{n+1 / 2}^{1 / 2}}-\frac{\hat{\xi}_{n+1 / 2}}{\hat{\ell}_{n+1 / 2}^{1 / 2}}-\frac{\hat{\xi}_{n-1 / 2}}{\hat{\ell}_{n-1 / 2}^{1 / 2}},
\end{aligned}
$$


where $u_{n}, v_{n}$ are deterministic terms. These equations have been discretized with a standard Euler scheme, as follows

$$
\begin{aligned}
& \hat{\ell}_{n+1 / 2}(T+d T)=\hat{\ell}_{n+1 / 2}+d T u_{n}+\sqrt{d T}\left(\tilde{\zeta}_{n+1}-\tilde{\zeta}_{n}\right) \\
& \hat{\ell}_{n+1 / 2}(T+d T)=\hat{\ell}_{n+1 / 2}+d T v_{n}+ \\
& \quad \sqrt{d T}\left(\frac{\tilde{\xi}_{n+3 / 2}}{\hat{\ell}_{n+3 / 2}^{1 / 2}}+\frac{\tilde{\xi}_{n+1 / 2}}{\hat{\ell}_{n+1 / 2}^{1 / 2}}-\frac{\tilde{\xi}_{n+1 / 2}}{\hat{\ell}_{n+1 / 2}^{1 / 2}}-\frac{\tilde{\xi}_{n-1 / 2}}{\hat{\ell}_{n-1 / 2}^{1 / 2}}\right),
\end{aligned}
$$

where all the quantities on the r.h.s. are calculated at time $T$, and $\tilde{\zeta}_{n}, \tilde{\xi}_{n}$ are gaussian random variables.

The integration time step $d T$ has been chosen as the minimum between $d \tau$ and $d T^{*}$, where $d \tau$ is a fixed time step, and $d T^{*}=\min _{n}\left(d T_{n+1 / 2}^{*}\right)$, where $d T_{n+1 / 2}^{*}$ is the extrapolated closure time of interval $(n+1 / 2)$. This criterion allows one to have no kink annihilation, or one single annihilation event per update. The former case occurs if $d T=d \tau$, while the latter occurs if $d T=d T_{n^{*}+1 / 2}$ (in which case kinks $n^{*}$ and $n^{*}+1$ annihilate).
[1] P. B. Canham, J. Theor. Biol 26, 61 (1970).

[2] W. Helfrich, Z. Naturforsch. 28c, 693 (1973).

[3] J. F. Marko and E. D. Siggia, Phys. Rev. E 52, 2912 (1995)

[4] P. Benetatos and E. Frey, Phys. Rev. E 67, 051108 (2003)

[5] O. Pierre-Louis, Phys. Rev. E 83, 011801 (2011)

[6] R. Lipowsky, 349, 475 (1991).

[7] R. Gallotti and O. Pierre-Louis, Phys. Rev. E 75, 031801 (2007)

[8] T. Auth and G. Gompper, Phys. Rev. E 88, 010701 (2013)

[9] A. Hemmerle, L. Malaquin, T. Charitat, S. Lecuyer, G. Fragneto, and J. Daillant, 109, 19938 (2012)

[10] R. Bruinsma, A. Behrisch, and E. Sackmann, Phys. Rev. E 61, 4253 (2000)

[11] K. Sengupta and L. Limozin, Phys. Rev. Lett. 104, 088101 (2010)

[12] T. R. Weikl, M. Asfaw, H. Krobath, B. Rozycki, and R. Lipowsky, Soft Matter 5, 3213 (2009).

[13] J. Brugués, B. Maugis, J. Casademunt, P. Nassoy, F. Amblard, and P. Sens, 107, 15415 (2010).

[14] Biophysical Journal 94, 1836 (2008).

[15] Biophysical Journal 89, 724 (2005).

[16] T. Le Goff, P. Politi, and O. Pierre-Louis, Phys. Rev. E 90, 032114 (2014).

[17] P. S. Swain and D. Andelman, Phys. Rev. E 63, 051911 (2001)

[18] W. Helfrich, Z. Naturforsch. A 33, 305 (1978).

[19] D. Zuckerman and R. Bruinsma, Phys. Rev. Lett. 74,
3900 (1995).

[20] R. Lipowsky, Phys. Rev. Lett. 77, 1652 (1996).

[21] B. G. Lorz, A.-S. Smith, C. Gege, and E. Sackmann, Langmuir 23, 12293 (2007).

[22] E. Reister-Gottfried, K. Sengupta, B. Lorz, E. Sackmann, U. Seifert, and A.-S. c. v. Smith, Phys. Rev. Lett. 101, 208103 (2008)

[23] T. Bihr, U. Seifert, and A.-S. c. v. Smith, Phys. Rev. Lett. 109, 258101 (2012)

[24] T. Speck and R. L. C. Vink, Phys. Rev. E 86, 031923 (2012)

[25] A. Kusumi, C. Nakada, K. Ritchie, K. Murase, K. Suzuki, H. Murakoshi, R. S. Kasai, J. Kondo, and T. Fujiwara, Annual Review of Biophysics and Biomolecular Structure 34, 351 (2005) pMID: 15869394.

[26] H. Delanoë-Ayari, P. Lenz, J. Brevier, M. Weidenhaupt, M. Vallade, D. Gulino, J. F. Joanny, and D. Riveline, Phys. Rev. Lett. 93, 108102 (2004).

[27] T. Le Goff, O. Pierre-Louis, and P. Politi, preprint, arXiv:1504.00853 (2015).

[28] P. C. Hohenberg and B. I. Halperin, Rev. Mod. Phys. 49, 435 (1977)

[29] J. W. Cahn and J. E. Hilliard, The Journal of Chemical Physics 28, 258 (1958).

[30] K. Kawasaki and T. Ohta, Physica A 116, 573 (1982).

[31] T. Kawakatsu and T. Munakata, Prog. Theor. Phys. 74, 11 (1985).

[32] L. Malaquin, T. T. Charitat, and J. Daillant, The European Physical J E 31, 285 (2010). 